\title{
Magnitude of Long-acting and Permanent Family Planning Practice and Its Factors Among Contraceptive Users: A Cross-sectional Study
}

Alemu Abebe Wondyfraw ( $\sim$ alemuabebe71@gmail.com )

World Health Organization Country Office for Ethiopia https://orcid.org/0000-0002-8240-4361

Nigussie Assefa Kassaw

Addis Ababa University School of Public Health

\section{Research}

Keywords: Prevalence, determinant factors, family planning use, reproductive age-group women, Yilmanadensa Woreda, West Gojam zone, Ethiopia

Posted Date: April 7th, 2021

DOI: https://doi.org/10.21203/rs.3.rs-394817/v1

License: (c) (i) This work is licensed under a Creative Commons Attribution 4.0 International License.

Read Full License 
1 Magnitude of Long-acting and Permanent Family Planning Practice and Its Factors among

2 Contraceptive Users: A Cross-sectional Study

3 Alemu Abebe Wondyfraw ${ }^{1}$, Nigussie Assefa Kassaw ${ }^{2}$

$4 \quad{ }^{1}$ World Health Organization (WHO), Addis Ababa, Ethiopia

5 Email: alemuabebe71@gmail.com, wondyfrawa@who.int

$6{ }^{2}$ School of Public Health, College of Health Science, Addis Ababa University, Addis Ababa,

$7 \quad$ Ethiopia

8 Email: nigussie19@gmail.com, nigussie.assefa@aau.edu.et 


\section{Abstract}

23 Background: Poor contraceptive utilization increases the risk of unintended pregnancies that could results in complications and mortalities among women and children. To circumvent these adverse health outcomes, long-acting and permanent contraceptive methods are the most effective methods. This study explored the magnitude and associated factors of long-acting and permanent family planning methods utilization among current family planning users largely on the rural women of Ethiopia.

Methods: The study was a facility-based cross-sectional study design. The respondents were randomly selected in Amhara region, Ethiopia. The data were entered into EPI-INFO version 7.0, cleaned, and analyzed in SPSS version 24. Descriptive statistics were done. Bivariate and multivariable logistic regression models were used to identify the factors associated with the utilization of long-acting and permanent family planning methods.

Results: The total numbers of respondents were 356. The prevalence of long-acting and permanent family planning methods among current family planning users was $32.3 \%$. The mean age of participants was 30.1 \pm 7.67 (SD) years. Almost all (94.4\%) were Orthodox Christian followers and most of the respondents (91.0\%) married. Nearly half of the respondents $(45.2 \%)$ were illiterate and above half of the respondents (55.3\%) were housewives. The odds of longacting and permanent family planning methods utilization were statistically significant among women aged 25-34 years [AOR=5.10; 95\% CI: $(1.48,17.59)]$, women who completed secondary education $[\mathrm{AOR}=4.16 ; 95 \% \mathrm{CI}:(1.32,13.10)]$, women who had a positive attitude $[\mathrm{AOR}=3.05$; 95\% CI: $(1.45,6.43)]$, and women satisfied with facility care [AOR=2.08; 95\% CI: $(1.01,4.31)]$. Common reasons for low use of long-acting and permanent family planning methods were preference for short-acting contraceptives, fear of side effects, and method misconception. Conclusion: The utilization of long-acting and permanent family planning method was low. Factors and common reasons for low utilization of long-acting and permanent family planning methods among women who were current family planning users were identified. Therefore, we suggest the need to provide continuous education and awareness creation towards long-acting and permanent family planning methods. Unequivocally, enabling work environments, staff service deliveries and client service satisfaction are paramount important improve the coverage. 
51 Keywords: Prevalence, determinant factors, family planning use, reproductive age-group

52 women, Yilmanadensa Woreda, West Gojam zone, Ethiopia

\section{Plain English summary}

54 Contraceptive is a birth controlling method that enables people to space or limit the number of 55 children. It works to prevent maternal morbidity and mortality, fetal death, low birth weight, 56 prematurity, and child death. Unintended pregnancies could result in complications and 57 mortalities among women and children due to poor contraceptive utilization. The birth 58 controlling methods are the most effective methods to avoid unintended pregnancies. This study 59 showed that contraceptive methods coverage and associated factors in the rural setting of 60 Ethiopia while studies that were done so far focused on urban areas. This study was conducted in 61 selected health facilities. We selected four health facilities randomly in Yilmanadensa Woreda, 62 Amhara region, Ethiopia. Respondents were selected during their health facility visit to gain 63 birth control services in the selected health facilities. Among women who were current 64 contraceptive users, only $32.3 \%$ were used long-acting and permanent contraceptive methods. 65 Age, educational status, attitude towards long-acting and permanent contraceptive methods, and service satisfaction during health facility visit were factors that affect long-acting and permanent

67 contraceptive methods utilization. Women who were in the medium age group, women who 68 completed secondary education, women who had a positive attitude towards long-acting and permanent family planning methods, and women who were satisfied with the facility care services were the most users of long-acting and permanent contraceptive methods. Generally,

71 this study indicated that the use of long-acting and permanent contraceptive methods was low 72 among participated women in this study. 


\section{Introduction}

75 Family planning enables people to space or limit the number of children. It works to prevent maternal morbidity and mortality, fetal death, low birth weight, prematurity, and child death(1).

77 One most associated with lack of family planning utilization is a higher number of unintended pregnancies occur due to a lack of family planning use. This may result in unsafe abortion and its threatening consequences on the lives of women and babies(2).

To circumvent this and further adverse health outcomes among women and children, long-acting

81 and permanent family planning methods (LAPMs) are the most effective methods(3).

82 LAPMs include intrauterine contraceptive devices (IUDs), implants, female sterilization, and vasectomy. The first two methods are long-acting reversible while the latter two are permanent

84 methods(4).

85 The magnitude of long-acting and permanent contraceptive methods utilization was higher than 8650 percent in the world though it was low in Africa(5).

87 Despite the highest total fertility rate in Ethiopian(6), LAPMs coverage was considerably low and resulted in high unintended pregnancy-related complications including death(7). Unequivocally, LAPMs is a proven intervention to end complications and mortality. It also 90 reduced clients facility visit and contraceptive misuse(8).

91 Therefore, understanding the current magnitude and important risk, factors relevant to utilization

92 of LAPMs are certainly essential. Most importantly, this study informs the rural context of 93 Ethiopia which is barely reported so far. The evidence generated in this study is relevant to 94 design strategies and improve utilization of LAPMs. 
101 Methods and materials

102 Study design and setting

103 We conducted a facility-based cross-sectional study design from September 2018 to June 2019.

104 The study area was-in Yilmanadensa Woreda, West Gojam Zone, Amhara Region, Ethiopia. The study area covered a rural population where primary health care services are the largest of all.

Sample size and sampling procedure

All reproductive age group women who had a current health facility visit for family planning service were included in the study. The sample size was 383 determined by single population proportion formula. We assumed $34.7 \%$ proportion(9), 95\% confidence level, 5\% margin of error, and $10 \%$ non-response rate. In the study district, there were eleven health centers and four of them were selected by simple random sampling method. Women who had a family planning visit in these selected four health centers were randomly chosen for an interview.

\section{Study variables}

114 Dependent variable: Long-acting and permanent family planning methods (LAPMs) use

115 Independent variables: age, education, religion, ethnicity, marital status, residence area, occupation, income, number of children and future birth intention, awareness about long-acting

117 and permanent family planning methods, knowledge on the duration of LAPMs to prevent 118 pregnancy, knowledge about the advantage of LAPMs, knowledge about the disadvantage of 119 LAPMs, knowledge about types of LAPMs, fear of side effects, perceptions about LAPMs, fear 120 of infection, fear of infertility, perception about the privacy issue, perception about services 121 procedure, perception about waiting time, LAPMs misconception Operational definitions

123 Utilization of LAPMs: reproductive-age women using at least one of the four long-acting and 124 permanent contraceptives, Intrauterine contraceptive devices, Implant, Vasectomy, Female 125 sterilization, during the study period(9).

126 Reproductive age women: women who were in the age group of 15-49 years and sexually 127 active(10). 
128 Family planning user women: reproductive age-women who were using any contraceptive methods including both modern and natural family planning methods during the study period(6). Knowledge of LAPMs: assessed using 20 key questions; the knowledge of respondents on the type of long-acting and permanent family planning methods, sources of information about LAPMs, advantage, and disadvantage of LAPMs use, source of LAPMs, duration of IUD, and implant to prevent pregnancy, aware of the return of fertility after removal of long-acting reversible contraceptives, and the knowledge on whether it is permanent or reversible methods. Then 20 questions were computed and labeled to good and poor knowledge based on the mean. The mean score was determined after computing 20 knowledge assessing questions(9).

Good knowledge LAPMs: Women who scored above the mean of the correct answers for questions set to assess the knowledge of respondents on LAPMs(11).

Poor knowledge of LAPMs: Women who scored below or equal to the mean score of the correct answers from knowledge assessing questions of LAPMs(11).

Attitude towards LAPMs: assessed using 20 Likert scale questions; acceptance of LAPMs by respondents, by religious institutions, and by the community, husband and family opinion, consequences of LAPMs; infertility, uterus cancer, ectopic pregnancy, irregular bleeding of menses, a decrease of sexual desire, infection, IUDs move out of the womb, interfere during sexual intercourse, prevent doing heavy works, nulliparous women have to use intrauterine contraceptive devices, insertion of intrauterine contraceptive devices loses privacy. Female sterilization and vasectomy decrease sexual desires were other points addressed to assess attitude to long-acting and permanent family planning methods. Then 20 questions were computed and categorized into a positive attitude and a negative attitude based on the mean. The mean score was determined after computing 20 attitude assessing questions(9).

151 Favorable Attitude towards LAPMs: Women who scored above the mean of the correct answers 152 for questions ready to measure the attitude of respondents towards LAPMs(11).

153 Unfavorable Attitude towards LAPMs: Women who scored less than or equal to the mean score 154 from attitude measuring LAPMs questions(11).

155 Service satisfaction: assessed using eight service satisfaction questions. The questions were how 156 were clients treated during their visit for family planning, how long clients did stay in health 
157 facilities, what did clients feel about waiting time, whether health providers ask them if they had 158 faced problems and they wanted to discuss the problems, did the health providers try to 159 understand the problem and give suggestions what clients should do. In addition to these, clients' 160 satisfaction with the treatments and advice during health facility visits for family planning 161 service was the other question. Then 8 questions were computed and categorized into service 162 satisfaction and service dissatisfaction based on the mean. The mean score was determined after 163 computing eight service quality satisfaction assessing questions(9).

164 Service satisfaction: Women who scored above the mean of the correct answers for questions 165 able to measure service satisfaction of respondents(11).

166 Service dissatisfaction: Women who scored less than or equal to the mean score from service 167 satisfaction assessing questions(11).

\section{Data processing and analysis}

169 We used a pre-tested structured questionnaire to collect the data. Trained female diploma nurses 170 collected the data supervised by a degree holder nurse. The data collection took place for 40 days 171 from 21 February to 31 March 2019. The supervisor edited and cleaned the data daily. The data 172 were entered into EPI-INFO version 7.0 and cleaned and analyzed in SPSS version 24. 173 Descriptive statistics such as frequency, percentage, means, and standard deviations were done. 174 Bivariate and multivariable logistic regression models were used to identify the factors 175 associated with the utilization of LAPMs. Factors with a p-value of less than 0.25 in bivariate 176 analysis were included in the multivariable logistic regression model to control confounders. The 177 level of association was detected through adjusted odds ratios with a 95\% confidence interval 178 and a p-value of less than 0.05 . 
185 Results

186 Socio-demographic characteristics

187 A total of 356 respondents participated in the study with a 93\% response rate. The study 188 participants' mean age was 30.1( \pm 7.67 SD) years. Almost all participants (94.4\%) were 189 Orthodox Christian followers and most (91.0\%) of them were married. Among the respondents, 190 nearly half of them (45.2\%) were illiterates, $(55.3 \%)$ were housewives, and (54.8\%) had less 191 than 500 Ethiopian Birr monthly income (Table 1).

192 Table 1 Socio-demographic characteristics of respondents, Amhara Region, Ethiopia, 2019

\begin{tabular}{lll}
\hline Variables & Frequency & Percentage (\%) \\
\hline Age & 93 & 26.1 \\
$15-24$ & 152 & 42.7 \\
$25-34$ & 111 & 31.2 \\
$35-49$ & & \\
Religion & 336 & 94.4 \\
Orthodox & 20 & 5.6 \\
Muslim & & \\
Marital status & 324 & 91.0 \\
Married & 22 & 6.2 \\
Separated & 10 & 2.8 \\
Divorced & & \\
Educational status of respondents & 161 & 45.2 \\
No formal education & 61 & 17.1 \\
Primary & 66 & 18.6 \\
Secondary & 68 & 19.1 \\
College and above & & \\
Husband educational status & 106 & 32.7 \\
No formal education & 60 & 18.5 \\
Primary & 68 & 21.0 \\
Secondary & 90 & \\
College and above & & \\
& &
\end{tabular}


Occupational status of respondents

Housewife

197

55.3

Self employee

112

31.5

Government employee

47

13.2

Husband occupational status

Farmer

135

41.7

Self employee

111

34.2

Government employee

78

24.1

Monthly income (ETB) of respondents

$\leq 500$

195

54.8

501-1000

53

$>1000$

108

30.3

Husband monthly income (ETB)

$\leq 500$

57

17.6

501-1000

60

18.5

$>1000$

207

63.9

Total monthly income (ETB) of the family

$\leq 500$

38

10.7

501-1000

54

15.2

$>1000$

264

74.1

Number of family size

$<5$

241

67.7

$\geq 5$

115

32.3

Residence environment

Rural

241

67.7

Urban

115 
196 Respondent's obstetrics characteristics

197 More than three-fourths of the respondents (77.5\%) had ever been pregnant. Of these, $(95.3 \%)$

198 had given birth. Age at first marriage ranged 4 to 27 years and the mean age of the first marriage

199 was $16.29 \pm 4.49$ (SD) years. The mean age of first pregnancy was 20.06 \pm 3.02 (SD) years and

200 ranged between 14 and 30 years. Age at first delivery ranged from 14 to 31 years old and the

201 mean age was 20.87 \pm 3.01 (SD) years. The mean number of children was 2.46 ranged from 2.29

202 to 2.63 , and most of the respondents $(82 \%)$ had future birth intension (Table 2).

203 Table 2 Respondents obstetrics characteristics, Amhara Region, Ethiopia, 2019

\begin{tabular}{lll}
\hline Variables & Frequency & Percentage (\%) \\
\hline Age at the time of first marriage & 106 & 31.0 \\
$\leq 14$ & 144 & 42.1 \\
$15-19$ & 82 & 24.0 \\
$20-24$ & 10 & 2.9 \\
$\geq 25$ & & \\
Age at the time of first pregnancy & 118 & 42.8 \\
$\leq 19$ & 135 & 48.9 \\
$20-24$ & 23 & 8.3 \\
$\geq 25$ & & \\
Age at the time of first birth & 82 & 31.2 \\
$\leq 19$ & 150 & 57.0 \\
$20-24$ & 31 & 11.8 \\
$\geq 25$ & & \\
Number of live children & 97 & 37.5 \\
$<3$ & 162 & 62.5 \\
$\geq 3$ & & 82.0 \\
Future birth intention & 292 & 18.0 \\
Yes & 64 & \\
No & & \\
\hline & & \\
\hline
\end{tabular}


205 Respondents LAPMs Knowledge

206 The majority (84\%) of the study participants ever heard about LAPMs and three-fourths (75\%)

207 of them knew at least one of the LAPMs. Implant was the commonly known one (98.9\%).

208 Among women who knew LAPMs, $68.2 \%$ of them understood LAPMs uses for child spacing.

209 Greater than half of the respondents (57.7\%) knew that LAPMs had bad health effects. Almost

210 all (91.8\%) of them knew that LAPMs were adequately available in their area and $92.1 \%$ knew

211 that LAPMs were free of charge. Most of the respondents (91.4\%) knew implant is reversible

212 while $33 \%$ of them knew that intrauterine contraceptive device (IUD) is also reversible (Table $2133)$.

214 Table 3 Respondents knowledge about LAPMs, Amhara Region, Ethiopia, 2019

\begin{tabular}{lll}
\hline Variable & Frequency & Percentage (\%) \\
\hline Ever heard about LAPMs & 299 & 84.0 \\
Source of information & & \\
Health extension & 217 & 72.6 \\
Health service provider & 155 & 51.8 \\
Friend & 112 & 37.5 \\
Neighbor & 102 & 34.1 \\
Media & 98 & 32.8 \\
Types of LAPMs the respondents know & & \\
IUD & 103 & 38.6 \\
Implant & 264 & 98.9 \\
Female sterilization & 29 & 10.9 \\
Vasectomy & 22 & 8.2 \\
Advantage of LAPMs & & \\
Child spacing & 182 & 68.2 \\
Prevent unwanted pregnancy & 157 & 58.8 \\
Limit family size & 129 & 48.3 \\
Prevent maternal and child death & 52 & 19.5 \\
Prevent abortion & 49 & 18.4 \\
Disadvantage of LAPMs & &
\end{tabular}


Health side effect

Infertility

Infection

Cancer

Source of LAPMs

Government health center

207

140

119

43

26

28

38

102

68

31

Duration of IUD to prevent pregnancy

$<3$ years

3-5 years

$5-10$ years

10- 12 years

$>12$ years
57.7

18.4

24.7

7.9

84.5

57.1

48.6

17.6

10.6

10.5

14.2

38.2

Duration of an implant to prevent pregnancy

$<$ 1year

$28 \quad 10.5$

1- 3 years

$51 \quad 19.1$

3- 5 years

179

25.5

11.6

$>5$ years

$\begin{array}{ll}9 & 3.4\end{array}$

Knowledge of LAPMs

Good

106

43.4

Poor

$138 \quad 56.6$ 
216 The study showed that all respondents had ever heard at least one family planning method 217 (Figure 1).

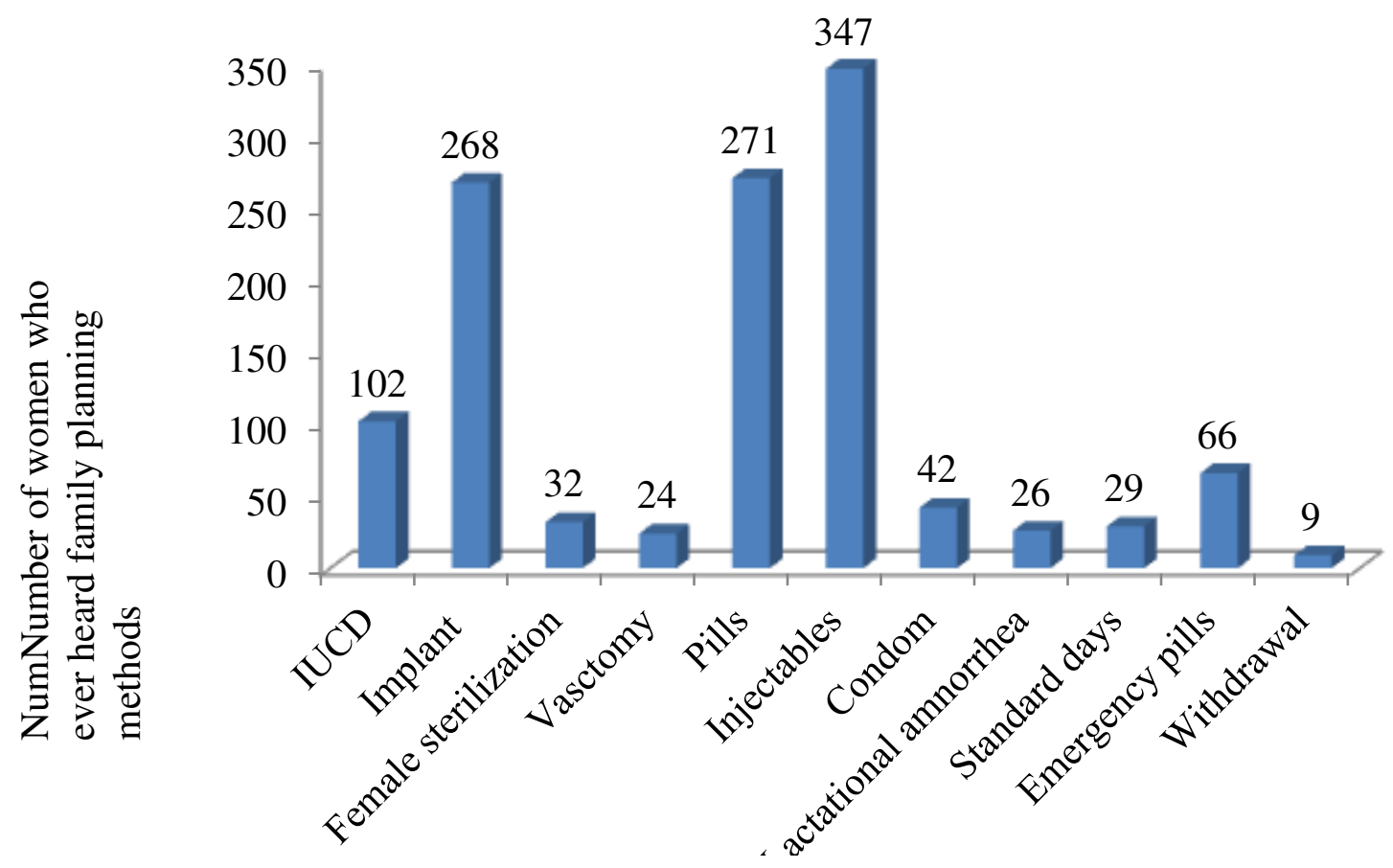

Types of ever heard family planning methods

218 Fig 1 Types of family planning methods respondent ever heard, Amhara Region, Ethiopia, 2019

219 Attitude and service quality perception towards LAPMs

220 The study showed (68.5\%) of respondents accepted LAPMs and (9.3\%) were not sure to accept 221 the LAPMs. Nearly one-fourth of the respondents (22.2\%) did not accept LAPMs, and (23.3\%) 222 of them believed that LAPMs decrease the sexual desire of users while more than half $(54.8 \%)$ 223 of respondents, perceived insertion of intrauterine contraceptive devices lose privacy. On the other 224 hand, a quarter (22.5\%) of respondents believed that female sterilization decreases the sexual 225 desire of users. Some of the total respondents (18.3\%) faced poor treatment in health facilities 226 during family planning service, and (19.1\%) of them faced too long waiting times (Table 4). 
228 Table 4 Respondent's attitude and service quality perception towards LAPMs, Amhara Region, 229 Ethiopia, 2019

\begin{tabular}{lll}
\hline Variables & Frequency & Percentage (\%) \\
\hline Attitude about LAPMs (356) & & \\
Positive attitude & 181 & 50.8 \\
Negative attitude & 175 & 49.2 \\
Satisfaction of treatment & & \\
Satisfied & 195 & 54.8 \\
Unsatisfied & 161 & 45.2 \\
LAPMS are acceptable by religious institutions & & \\
Strongly agree & 9 & 2.5 \\
Agree & 22 & 6.2 \\
Not sure & 38 & 10.7 \\
Disagree & 133 & 37.3 \\
Strongly disagree & 154 & 43.3 \\
Husbands had a positive attitude to LAPMS & & \\
Strongly agree & 45 & 12.7 \\
Agree & 120 & 33.7 \\
Not sure & 63 & 17.7 \\
Disagree & 83 & 23.3 \\
Strongly disagree & 146 & 12.6 \\
Families had a positive attitude towards LAPMS & & \\
Strongly agree & 50 & 14.0 \\
Agree & 106 & 29.8 \\
Not sure & 51 & 30.4 \\
Disagree & 108 & 11.5 \\
Strongly disagree & 41 & \\
LARCs does not cause infertility & & \\
Strongly agree & 33.3 \\
Agree & 117 \\
Not sure & & \\
& & \\
\hline
\end{tabular}


Disagree

Strongly disagree

LAPMs does not cause uterus cancer

Strongly agree

Agree

Not sure

131

36.8

Disagree

Strongly disagree

LAPMs does not cause ectopic pregnancy

Strongly agree

Agree

Not sure

180

50.6

Disagree

Strongly disagree

\section{LAPMs does not cause irregular bleeding}

Strongly agree

Agree

16

54

55

141

90

39.6

Strongly disagree

LAPMs does not decrease sexual desire

Strongly agree

9.6

Agree

141

Not sure

63

20

25.3

Disagree

Strongly disagree

\section{LAPMs does not cause infection}

Strongly agree

33

73

9.0

Agree

146

20.2

Not sure

72
41.0

20.5 
Strongly disagree

IUD do not move out of the womb

Strongly agree

Agree

Not sure

Disagree

Strongly disagree

IUD insertion do not lose privacy

Strongly agree

44

Agree

Not sure

Disagree

Strongly disagree

33

85

34

20

51

66

89
184

106

Female sterilization does not decrease sexual

desire

Strongly agree

Agree

35

90

151

54

26

Strongly disagree

Vasectomy does not make the penis impotence

Strongly agree

Agree

Not sure

Disagree

Strongly disagree
33

76

189

44

14
9.3

9.6

5.6

29.8

230 Long-acting and permanent contraceptive methods utilization

231 Among the respondents, 43.3\% had ever used LAPMs. Almost all (94.8\%) of them had ever

232 used an implant. On the other hand, a small number of respondents $7.1 \%$ and $2 \%$ had ever used 
233 IUD and female sterilization respectively. The mean respondent age to start LAPMs was $23424.99 \pm 5.44$ years.

235 The prevalence of LAPMs was 32.3\% [95\% CI; $(27.5,37.1)]$, implying only one-third of 236 respondents were current users of LAPMs. Most of the respondents (90.4\%) used implant. Some 237 of them (7\%) were using IUD and only (2.6\%) women were using female sterilization (Figure 2).

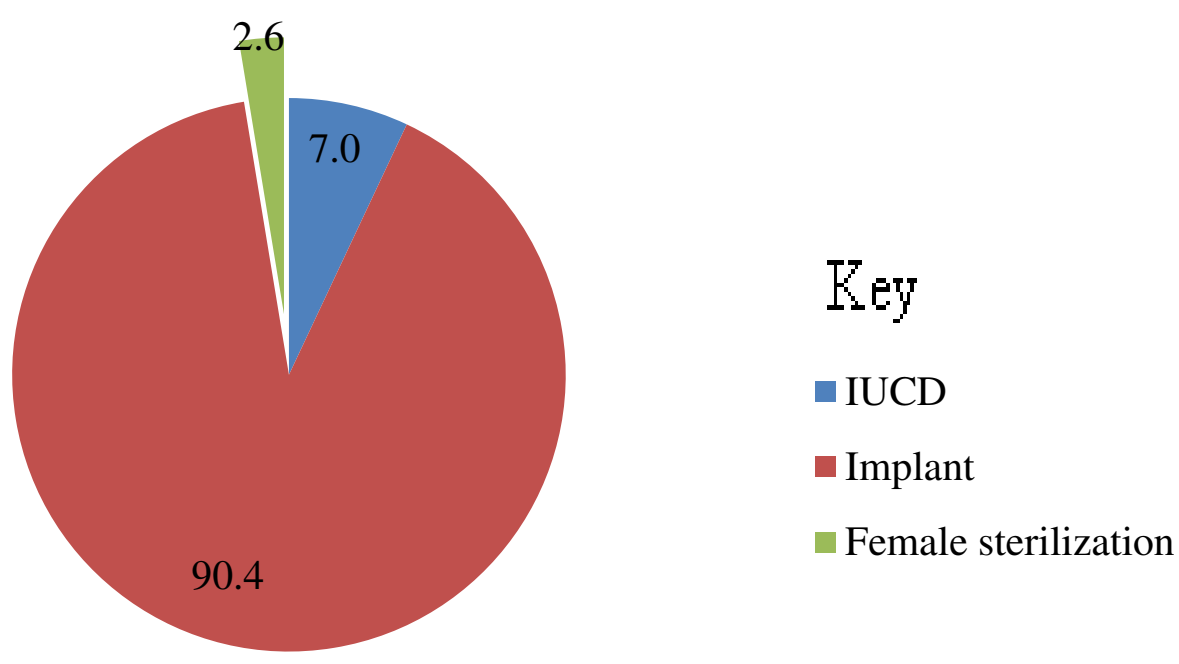

\section{Percentage}

239 Fig 2 Respondents' current use of LAPMs, Amhara Region, Ethiopia, 2019

240 The main reasons for LAPMs utilization were the benefit of LAPMs over other contraceptives, 241 child spacing, and advice from health professionals. On the other hand, the barriers to use 242 LAPMs were preference for short-acting contraceptives, fear of side effects, and method 243 misconception.

244 Among respondents who were using LAPMs, 37.0\% of women had faced health problems due to 245 the utilization of LAPMs (Figure 3). 


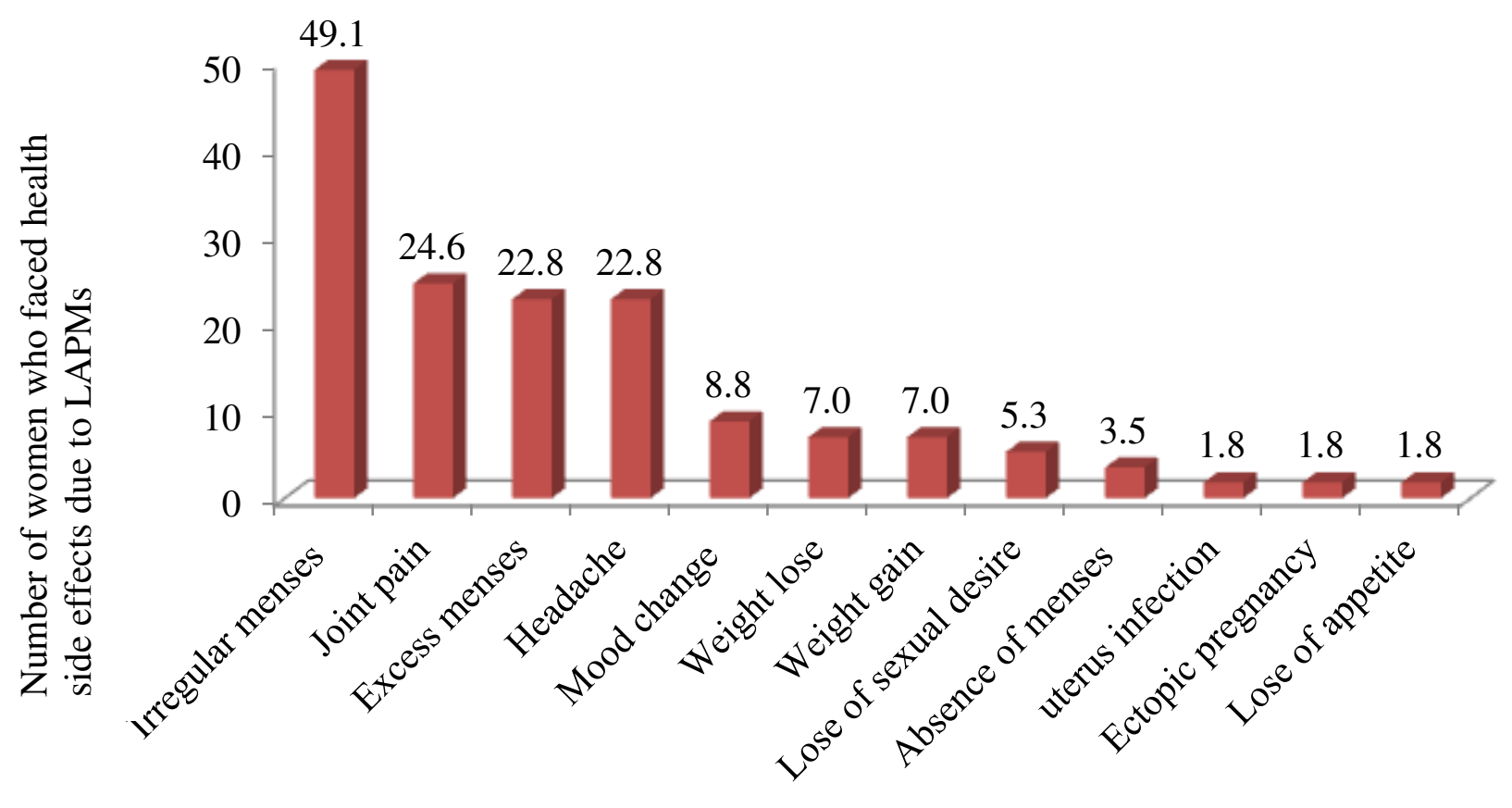

Health side effect of LAPMs

247 Fig 3 Reported health side effects of LAPMs among respondents who were on LAPMs, Amhara

248 Region, Ethiopia, 2019

\section{Factors associated with LAPMs utilization}

250 Age, education, attitude, and service satisfaction had statistically a significant association with 251 LAPMs utilization. The odds of LAPMs utilization among women aged 25-34 years were five 252 times higher compared to $15-24$ years age group [AOR=5.10; 95\% CI: $(1.48,17.59)]$. The odds 253 of LAPMs among women who completed secondary education were four times greater than 254 women who had no formal education [AOR=4.16; 95\% CI: $(1.32,13.10)]$. The odds of LAPMs

255 among women who had a positive attitude were three times greater than women who had a 256 negative attitude $[\mathrm{AOR}=3.05 ; 95 \% \mathrm{CI}:(1.45,6.43)]$, and the odds of LAPMs among women who 257 satisfied with facility care were two times higher compared to their dissatisfied counterparts $258 \quad[$ AOR $=2.08 ; 95 \%$ CI: $(1.01,4.31)]$ (Table 5). 
261 Table 5 Factors associated with LAPMs, Amhara Region, Ethiopia, 2019

\begin{tabular}{|c|c|c|c|c|}
\hline \multirow[b]{2}{*}{ Determinant variables } & \multicolumn{2}{|c|}{ LAPMs utilization } & \multirow[b]{2}{*}{ COR $(95 \% \mathrm{CI})$} & \multirow[b]{2}{*}{$\operatorname{AOR}(95 \% \mathrm{CI})$} \\
\hline & Yes & No & & \\
\hline \multicolumn{5}{|l|}{ Age } \\
\hline $15-24$ & 16 & 77 & 1 & 1 \\
\hline $25-34$ & 62 & 90 & $3.32(1.77,6.21)^{*}$ & $5.10(1.48,17.59) * *$ \\
\hline $35-49$ & 37 & 74 & $2.41(1.23,4.69)^{*}$ & $2.82(0.74,10.77)$ \\
\hline \multicolumn{5}{|l|}{ Education } \\
\hline No formal education & 34 & 127 & 1 & 1 \\
\hline Primary & 17 & 44 & $1.44(0.73,2.84)$ & $1.97(0.69,5.63)$ \\
\hline Secondary & 33 & 33 & $3.74(2.02,6.90) *$ & $4.16(1.32,13.10)^{* * *}$ \\
\hline College and above & 31 & 37 & $3.13(1.70,5.75) *$ & $1.17(0.35,3.88)$ \\
\hline \multicolumn{5}{|l|}{ Attitude of LAPMs } \\
\hline Positive & 90 & 91 & $5.93(3.55,9.92) *$ & $3.05(1.45,6.43)^{* *}$ \\
\hline Negative & 25 & 150 & 1 & 1 \\
\hline \multicolumn{5}{|l|}{ Service satisfaction } \\
\hline Satisfied & 75 & 120 & $1.89(1.19,2.99)^{*}$ & $2.08(1.01,4.31)^{* *}$ \\
\hline Dissatisfied & 40 & 121 & 1 & 1 \\
\hline \multicolumn{5}{|l|}{ Number of live children } \\
\hline$\geq 3$ & 61 & 101 & $1.57(0.91,2.70)$ & $1.92(0.80,4.62)$ \\
\hline$<3$ & 27 & 70 & 1 & 1 \\
\hline \multicolumn{5}{|l|}{ Knowledge of LAPMs } \\
\hline Good & 53 & 53 & $1.38(0.83,2.3)$ & $1.49(0.71,3.11)$ \\
\hline Poor & 58 & 80 & 1 & 1 \\
\hline \multicolumn{5}{|l|}{ Residence } \\
\hline Urban & 47 & 68 & $1.76(1.10,2.80) *$ & $0.86(0.37,2.01)$ \\
\hline Rural & 68 & 173 & 1 & 1 \\
\hline \multicolumn{5}{|l|}{ Monthly income } \\
\hline$\leq 500 \mathrm{ETB}$ & 58 & 137 & 1 & 1 \\
\hline 501- 1000 ETB & 13 & 40 & $0.77(0.38,1.54)$ & $0.88(0.31,2.52)$ \\
\hline$>1000 \mathrm{ETB}$ & 44 & 64 & $1.62(0.99,2.66)$ & $1.11(0.41,3.01$ \\
\hline
\end{tabular}




\section{Future birth intention}

\begin{tabular}{lllll} 
No & 16 & 48 & $0.65(0.35,1.20)$ & $1.03(0.40,2.66)$ \\
Yes & 99 & 193 & 1 & 1 \\
\hline
\end{tabular}

$262 * *$ Age group of 25-34 $(\mathrm{p}=0.01)$, secondary education $(\mathrm{p}=0.015)$, positive attitude $(\mathrm{p}=0.003)$

263 and satisfied with the service $(\mathrm{p}=0.049)$

264

265

266

267

268

269

270

271

272

273

274

275

276

277

278

279

280

281 
282 Discussion

283 The study found a low proportion (32.3\%) of LAPMs utilization. The finding concurs with other 284 studies in Ethiopia $(9,12,13)$. The study settings and design could contribute to the consistency 285 of the findings.

286 However, it was significantly lower than the finding from Gambia(14). The study in Gambia was conducted in a single facility and had a smaller sample. Also unlike our study, Depo-Provera was considered as LAPMs in Gambia's study upsurge the magnitude. Likewise, there are also other studies in Ethiopia(4, 8, 15, 16, 17) that are contrary to our finding. We assumed study time and setting as well as sampling methods and sample size contributed to the difference.

Majority of the respondents had awareness about LAPMs unlike studies reported in Mekelle town(18), Debre-Markos town(19), Arbaminch town(8) and Goba town(20) of Ethiopia. We anticipated that source of information took the possible reason for the variation as the study settings varied.

295 Government health facilities were the main LAPMs providers in this study similar to other 296 studies in Ethiopia $(4,17,18)$. The methodology and study settings were similr to our study that 297 could be the possible reason of similarity.

298 Implant was commonly used contraceptive followed by IUD and female sterilization in this 299 study. Other studies in Ethiopia also showed a similar result $(9,12,13,17,18)$. We assumed the sample size and study design had a contribution to the sameness of the findings.

301 The main barriers of LAPMs utilization were the tendency of short-acting contraceptives over 302 LAPMs, fear of side effects, and method misconception which was similar to a study conducted in Debre-Berhan(11). We assumed that the sampling technique contributed to the consistency.

304 The odds of LAPMs utilization among women in the age group of 25-34 years were five times 305 higher as compared to $15-24$ years of age $[\mathrm{AOR}=5.10 ; 95 \% \mathrm{CI}$ : $(1.48,17.59)]$. Correspondingly, 306 a similar finding was reported in Jinka town(4). The study design and setting could have a role of 307 similarity of the findings. But, another study in Addis Ababa reported the opposite(21). We 308 anticipated that the study setting took the reason of inconsistency as younger women who live in 309 Addis Ababa could have more information than their counterparts in a rural community. 
310 The odds of LAPMs utilization among women who completed secondary education were four 311 times higher than women who had no formal education [AOR=4.16; 95\% CI: $(1.32,13.10)]$.

312 Similar findings were reported in Gondar city(9), Bati town(12), and Nekemte town(22). The

313 sample size could have a contribution to the consistency. On the other hands, few studies in Jinka 314 town(4), Adigrat town(10), and Addis Ababa(21) in Ethiopia reported dissimilar findings. We 315 assumed the study period, study settings, and sampling technique may cause discrepancies.

316 The odds of LAPMs utilization among women who had a positive attitude towards LAPMs were 317 three times higher compared to women who had a negative attitude [AOR=3.05; 95\% CI: (1.45, 318 6.43)]. Findings in Debre-Berhan(11) and Bati town(12) were consistent with this result. We 319 assumed that the study settings may input to similarity of the findings.

320 The odds of LAPMs utilization among women who were satisfied with treatment during their 321 visit to health facilities were twice higher than dissatisfied women [AOR=2.08; 95\% CI: (1.01, 322 4.31)]. It was similar to a finding in Debre-Berhan, Ethiopia(11). We assumed that the study 323 setting may have a role in the consistency of the findings.

Conclusion

325 Long-acting and permanent family planning methods utilization was low. Age, educational 326 status, attitude towards LAPMs, and service satisfaction were statistically significantly 327 associated with LAPMs utilization. Also, common barriers reported for LAPMs utilization were the type of contraceptive preference, fear of side effects, and method misconception.

329 Abbreviations

330 EDHS: Ethiopian Demographic and Health Survey, FP: Family Planning, HC: Health Center, 331 IUD: Intrauterine Contraceptive Device, LAPMs: Long-acting and Permanent Contraceptive 332 Methods, ETB: Ethiopian Birr

\section{Declarations}

\section{Ethics approval and consent to participate}

335 Ethical clearance was obtained from the Ethical Review Committee of the School of Public 336 Health, Addis Ababa University. Permission letters were obtained from each administrative body and health facilities. Study participants were informed about the study objective and the right not 
338 to take part or withdraw participation before the interview started or at any time during the 339 interview. For all respondents, written consent was secured, and the respondent's information 340 was completely confidential.

\section{Consent for publication}

342 Not applicable

343 Availability of data and materials

344 The dataset for this study is available from the correspondent author and can be provided on a 345 reasonable request.

346 Competing interests

347 The authors declared that they have no competing interests.

\section{$348 \quad$ Funding}

349 No financial support from any organization.

350 Authors' contributions

351 AAW conceived the study, designed the study, developed the tool, coordinated data collection, 352 performed the statistical analysis, interpreted the findings and drafted the manuscript.

353 NAK designed the study, developed the tool, performed the statistical analysis interpreted the 354 findings, guided the project, and drafted the manuscript. All authors read and approved the 355 manuscript.

356 Acknowledgment

357 We want to appreciate the study participants, the data collectors, and the supervisor for their 358 committed participation in the data collection. 
364 References

365 1. Jacobstein R. Long-acting and permanent contraception: an international development, service

366 delivery perspective. Journal of midwifery \& women's health. 2007;52(4):361-7.

367 2. Tsui A, Brown W, Li Q. Contraceptive practice in sub-Saharan Africa. Population and development review. 2017;43 (Suppl 1):166.

3. Mesfin YM, Kibret KT. Practice and Intention to use long-acting and permanent contraceptive methods among married women in Ethiopia: Systematic meta-analysis. Reproductive health. 2016;13(1):78.

4. Mekonnen G, Enquselassie F, Tesfaye G, Semahegn A. Prevalence, and factors affecting the use of long-acting and permanent contraceptive methods in Jinka town, Southern Ethiopia: a cross-sectional study. Pan African Medical Journal. 2014;18(1):8.

375 5. United Nations, Department of Economic and Social Affairs, Population Division (2015).

376 Trends in Contraceptive Use Worldwide 2015 (ST/ESA/SER.A/349).Available 377 fromwww.unpopulation.org.Accessed 2015.

378 6. Central Statistical Agency (CSA) [Ethiopia] and ICF. 2016. Ethiopia Demographic and 379 Health Survey 2016. Addis Ababa, Ethiopia, and Rockville, Mary land, USA: CSA and ICF. 380 Available from www.DHSprogram.com. Accessed August 2016.

381 7. Kesetebirhan A. National Guideline for Family Planning Services in Ethiopia. The Federal 382 Democratic Republic of Ethiopia, Ministry of Health. 2011. Available from http://sistersources.worldbank.org/datastatisctics/resources/GNIPC.Accessed 2011.

8. Godana W, Wondmu F, Temesgen G, Timer G, Yesuf J, Habte M, et al. Utilization of longacting and permanent family planning methods among women's visiting family planning clinic in Arba Minch Hospital, Ethiopia. J Healt. Med Nur. 2015;15(1):62-71. acting and permanent contraceptive methods among women who have decided not to have more children in Gondar city. BMC women's health. 2017;17(1):75.

10. Gebremariam A, Addissie A. Intention to use long-acting and permanent contraceptive methods and factors affecting it among married women in Adigrat town, Tigray, Northern 392 Ethiopia. Reproductive health. 2014;11(1):24.

393 11. Asegidew W, Tariku B, Kaba M, Getachew S, Ketsela K. Acceptance and Positive Attitude 394 Increased Utilization of Long-acting and Permanent Family Planning Methods Among 
395 Reproductive Age Group Women from Debre Berhan District, Ethiopia: Quantitative and

Qualitative Analysis. J Community Med Health Educ. 2017;7(541):2161-0711.

12. Biza N, Abdu M, Reddy P. Long-acting reversible contraceptive use and associated factors among contraceptive users in the Amhara region, Ethiopia. A community-based cross-sectional study. Medico Research Chronicles. 2016;14(6):12.

13. Mota K, Reddy S, Getachew B. Unmet need for long-acting and permanent family planning methods among women in the reproductive age group in shashemene town, Oromia region, Ethiopia: a cross-sectional study. BMC women's health. 2015;15(1):51.

14. Anyanwu M, Alida BWN. Uptake of long-acting reversible contraceptive devices in the Western region of The Gambia. African health sciences. 2017;17(2):409-17.

15. Tekelab T, Melka AS, Wirtu D. Predictors of modern contraceptive methods use among married women of reproductive age groups in Western Ethiopia: a community based crosssectional study. BMC women's health. 2015;15(1):52.

16. Gebremeskel F, Getahun D, Kanko T, Tilahun A, Endrias B, Alamirew B, et al. Prevalence of modern contraceptive utilization and associated factors among women of reproductive age group at Boditi Town, Wolayita Zone, SNNPR, Ethiopia. American Journal of Nursing Science. 2017;6(6):447.

17. Yalew SA, Zeleke BM, Teferra AS. Demand for long-acting contraceptive methods and associated factors among family planning service users, Northwest Ethiopia: a health facilitybased cross-sectional study. BMC research notes. 2015;8(1):29.

18. Alemayehu M, Belachew T, Tilahun T. Factors associated with the utilization of long-acting and permanent contraceptive methods among married women of reproductive age in Mekelle town, Tigray region, north Ethiopia. BMC pregnancy and childbirth. 2012;12(1):6.

19. Bulto GA, Zewdie TA, Beyen TK. Demand for long-acting and permanent contraceptive methods and associated factors among married women of the reproductive age group in Debre Markos town, North West Ethiopia. BMC women's health. 2014;14(1):46.

20. Takele A, Degu G, Yitayal M. Demand for long-acting and permanent methods of contraceptives and factors for non-use among married women of Goba town, Bale Zone, South east Ethiopia. Reproductive health. 2012;9(1):26.

21. Teshome G, Woldeyohanis F, Deyessa N. Long-acting and Permanent Contraceptive Use in Arada Sub City, Addis Ababa, Ethiopia, 2017. J Community Med Health Educ. 2018;18(1):61. 
426 22. Melka AS, Tekelab T, Wirtu D. Determinants of long-acting and permanent contraceptive

427 methods utilization among married women of reproductive age groups in western Ethiopia: a 428 cross-sectional study. Pan African Medical Journal. 2015;22(1):10.

429

430

431

432

433

434

435

436

437

438

439

440

441

442

443

444

445

446 
Figures

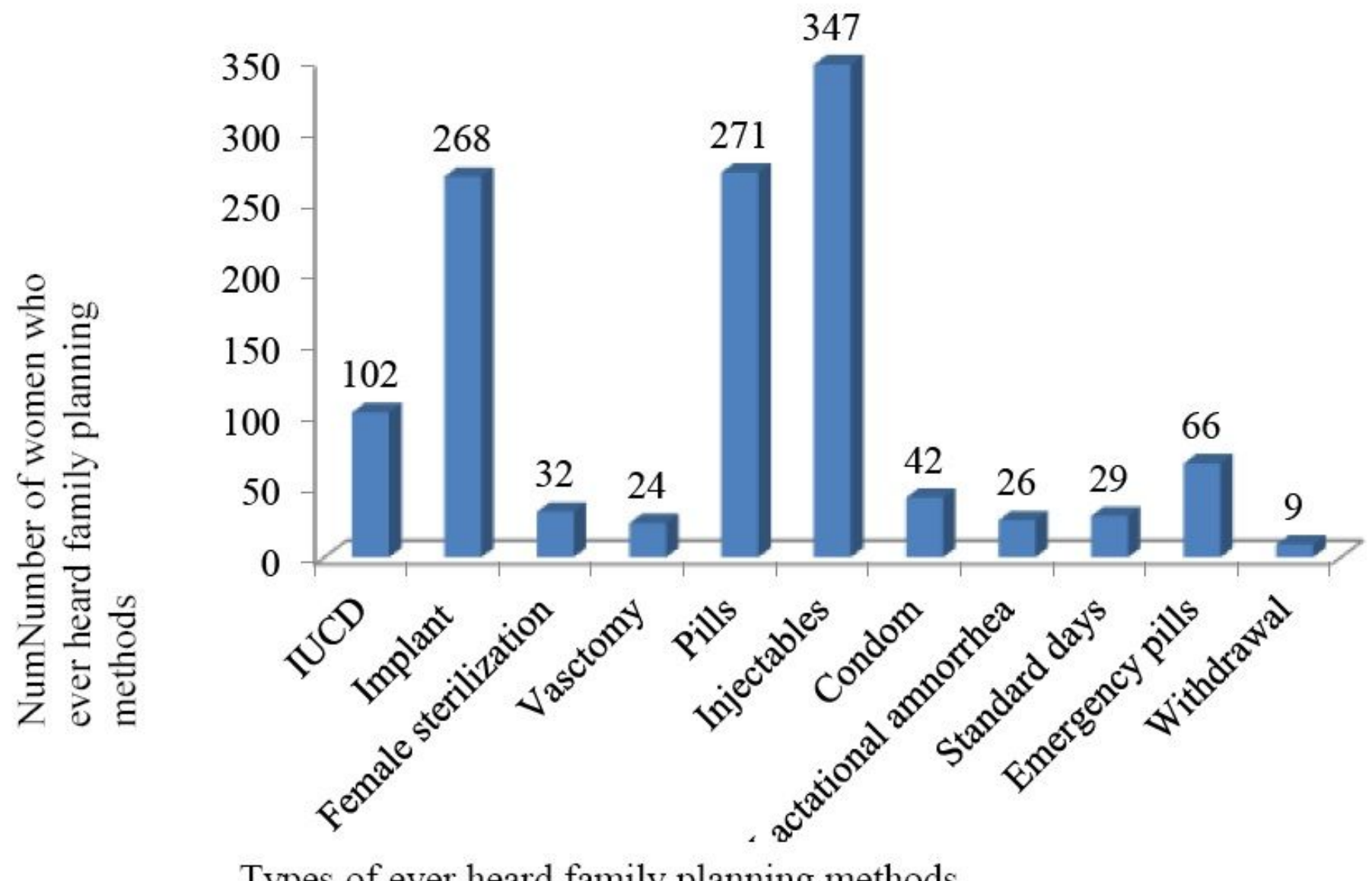

Types of ever heard family planning methods

Figure 1

Types of family planning methods respondent ever heard, Amhara Region, Ethiopia, 2019 


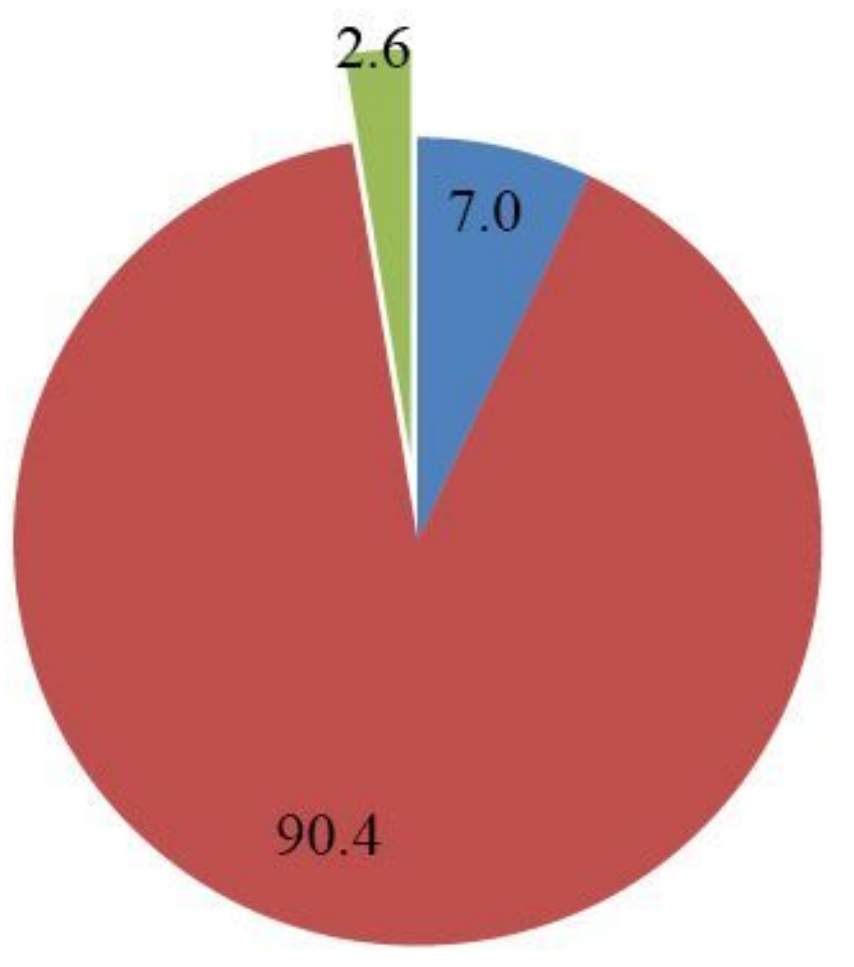

\section{Percentage}

\section{Figure 2}

Respondents' current use of LAPMs, Amhara Region, Ethiopia, 2019

\section{Key}

- IUCD

- Implant

Female sterilization 


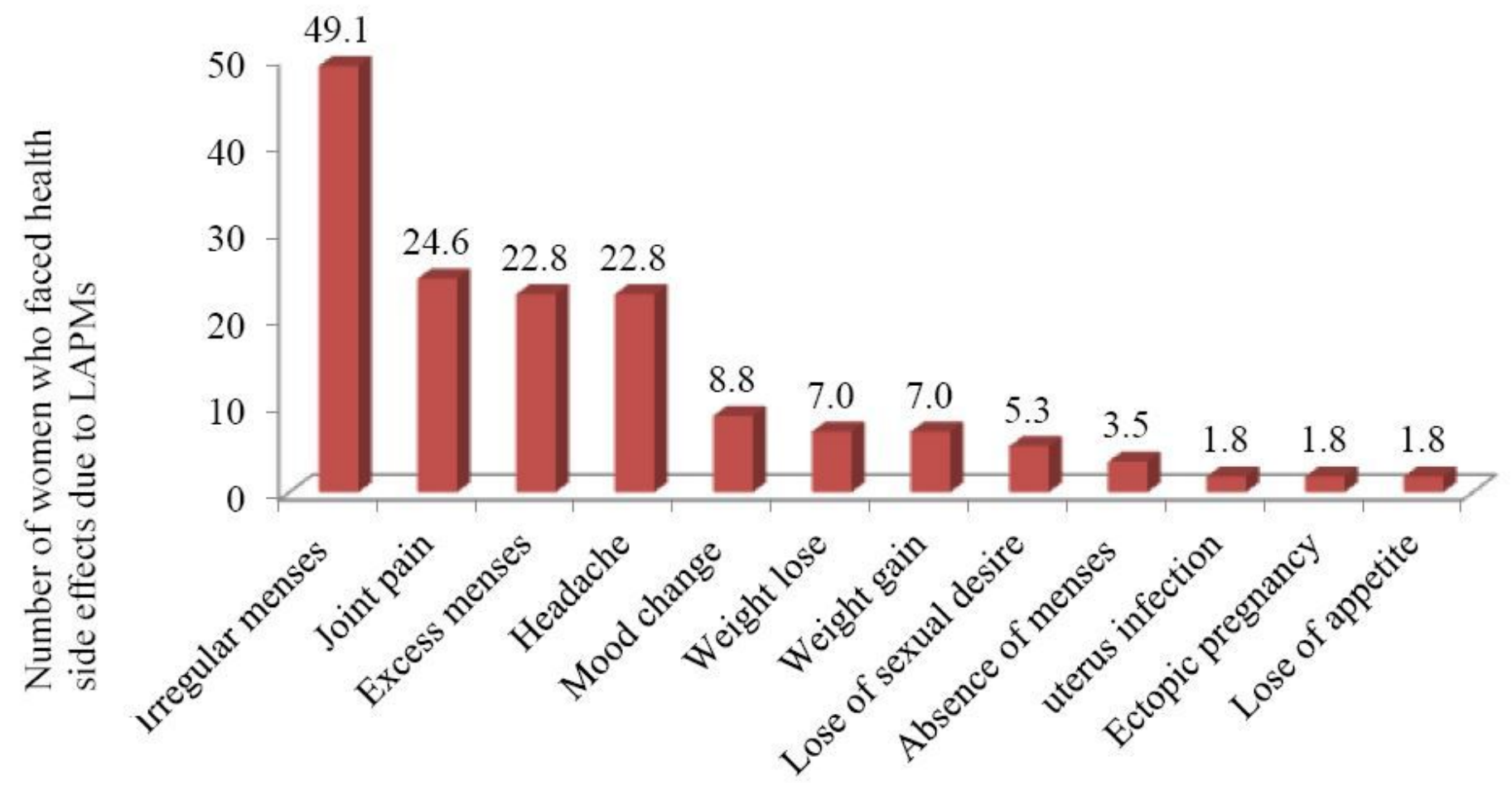

Health side effect of LAPMs

\section{Figure 3}

Reported health side effects of LAPMs among respondents who were on LAPMs, Amhara Region, Ethiopia, 2019 